immigrant). Compulsory admission to mental hospital would serve as evidence of an attack of 'insanity' but the effect of such an admission would depend on the patient's recovery.

Thus, contrary to popular psychiatric belief, it appears that compulsory admission may, if it produces a more rapid recovery, actually help a patient to obtain a US visa.

Highcroft Hospital

Nick Craddock

Highcroft Road

Erdington

Birmingham B23 6AX

\section{Doll phobia revisited}

SIR: It was very interesting to read Hatcher's description of successful exposure treatment of a doll phobia in a 14-year-old boy (Journal, August 1989, 155, 255257). He notes the rarity of doll phobia despite the ubiquity of dolls in most cultures. Such discordance between frequency of contact with stimuli and fear of them may be partly explained by a prepotency and preparedness of certain cues to arouse fear more than others do in a species as a result of its evolutionary history. Incidentally, the credit for the first description of doll phobia probably goes to Rangell (1952), cited by Marks (1987, p. 396).

\section{Institute of Psychiatry \\ De Crespigny Park \\ Denmark Hill \\ London SE5 8AF}

ISAAC MARKS

Reference
MARks, I. M. (1987) Fears, Phobias and Rituals. New York: Oxford
University Press.
RANGELL, L. (1952) The analysis of a doll phobia. International
Journal of Psychoanalysis, 33, 43.

\section{Historical melancholia}

SIR: Berrios's (1988) conceptual history of melancholia and depression, while scholarly and deep, is, I would suggest, in parts overstated. He says, for example, that the concept of bipolar disease required a series of conditions that were only met after 1950 . Certainly earlier physicians spoke at the least of the continuity of melancholia and mania, for example Boerhaave (1728), Morgagni (1761), and Esquirol (1812) (see Frosch, 1987). Furthermore, it appears clear that the notion of alternating disturbed states of slow, exerted behaviour with fast, even frantic, behaviour can be seen to have entered the popular imagination. Caldwell (1972) has drawn our attention to the fact that the final movement of the sixth of the Opus 18 quartets (written in 1799) was entitled by Beethoven "La Malinconia". The movement consists of six alternating slow and fast sections, the slow sections inhibited, unelaborated, the fast sections bristling with energy, leaps and trill-like figures. That Beethoven recognised what he was portraying is confirmed in a later letter to a friend about another piece contemporaneous with "La Malinconia": "everyone at that time sensed ... the mental state of melancholia and its phases". That this notion was not new in 1800 can also be seen in Pondourus's description (fourth century AD) that mania is "an intermittent disease which proceeds through a periodical variant ... it repeats itself once a year or more often ... melancholia occurs in autumn, whereas mania in summer" (source of quote unlocated).

Good, careful observation is not a recent invention. While theory can determine what we are aware of seeing, through the millennia family and friends of individuals with manic-depressive disease must have been aware of its alternation, its circularity, aware of each phase's characteristic thought contents, feeling states, and activity patterns, aware of the continuity between the phases. The shamans and physicians involved in their care must also have known these facts. We should not delude ourselves that our unassisted senses and reasoning powers are better than those of our ancestors. Changing social structure and new data, real or imagined, lead to newly organised observations and to new explanations, to new theories. Berrios's paper properly directs us to this level of historical inquiry.

Department of Psychiatry

William A. Frosch

Cornell University Medical College

1300 York Avenue

New York, NY 10021

USA

\section{References}

Berrios, G. E. (1988) Melancholia and depression during the 19th century: a conceptual history. British Journal of Psychiatry. 153, 298-304.

CaldWell, A. E. (1972) La Malinconia: Final movement of Beethoven's quartet op. 18, no. 6: a musical account of manic depressive states. Journal of the American Medical Association, 27, 241-248.

Frosch, W. A. (1987) Moods, madness, and music. I. Major affective disease and musical creativity. Comprehensive Psychiatry, 28, 315-322.

\section{ECT in old age}

SIR: We read with interest the paper by Benbow on electroconvulsive therapy (ECT) for depressive 
illness in old age (Journal, August 1989, 155, 147152). We also studied the use of ECT in elderly depressive patients (Malcolm \& Peet, 1989), but reached somewhat different conclusions.

Evidence is far from conclusive that ECT is the most effective form of treatment for elderly depressed patients. There are few, if any, methodologically satisfactory controlled trials of ECT conducted specifically in elderly populations. Available data from open, uncontrolled trials reveal widely varying response rates, with short-term response rates being much more favourable than longterm rates. This is in keeping with controlled trials in younger patients, showing ECT to have shortterm efficacy only (Johnstone et al, 1980; Brandon et al, 1984). Depression is a chronic disorder, with prolonged morbidity in the elderly, as Benbow rightly points out. The short-term nature of the ECT response necessitates continuation treatment with antidepressants after ECT to prevent relapse. If the clinician recommends ECT and fails to seek an initial effective antidepressant, there is no way of knowing which drug will be effective after ECT.

Furthermore, trials comparing ECT with antidepressant drugs also suffer from methodological defects (Rifkin, 1988) and do not take into account advances in psychopharmacology, such as the use of combined antidepressants and neuroleptics in delusional depression.

With regard to safety, ECT is held to be superior when compared with older antidepressants. However, as Benbow indicates, the cardiovascular side-effects of ECT are not uncommon. Trials comparing the relative safety of ECT and the newer antidepressants, known to be considerably less cardiotoxic than the older antidepressants, have yet to be done.

Finally, end-point states of depression requiring ECT are not reached suddenly. The duration of depressive symptoms may be especially long in the elderly, and in under-resourced services it is often only those who have deteriorated into severe depressive states who are admitted for treatment, by which time ECT may be considered inevitable. A comprehensive community-based service, which can mobilise pharmacological, psychological, and social help at an early stage of the depressive disorder, may well reduce the need for ECT.

Department of Psychiatry

Katy Malcolm Malcolm PeEt Northern General Hospital

Herries Road

Sheffield S5 $7 \mathrm{AU}$

\section{References}

Brandon, S., Cowley, P., McDonald, C., et al (1984) Electroconvulsive therapy: results from the Leicestershire trial. British Medical Journal, 288, 22-25.

Johnstone, E. C., DeAkIN, J. F. W., LAWLer, P., et al (1980) The Northwick Park electroconvulsive therapy trial. Lancet, ii, 1317-1320.

MalColm, K. \& PeEt, M. (1989) The use of electroconvulsive therapy in elderly depressive patients. In Antidepressants in the Elderly (ed. K. Ghose). Beckenham: Croom Helm.

RIFKIN, A. (1988) ECT versus tricyclic antidepressants: a review of the evidence. Journal of Clinical Psychiatry. 49, 3-7.

\section{Pimozide in pathological jealousy}

SIR: Byrne \& Yatham (Journal, August 1989, 155, 249-251) claim that treatment with pimozide was successful but produce no evidence. They are aware that pathological jealousy can occur in relation to alcoholism and one would assume that following admission to hospital this patient would have ceased to drink. In that case one would have expected the symptoms to have subsided in the ensuing few weeks without the use of any drugs; indeed the correct treatment of such a patient after admission would be not to give any drugs for some weeks and to review the diagnosis after that time if symptoms persisted. If persisting symptoms then subsided following the administration of a drug, that would be reasonably convincing evidence of the drug's effectiveness; the case report does not state that the patient was managed in this way.

I am bound to comment on the authors' statement that it is "well established" that pimozide is "very effective" in the treatment of monosymptomatic hypochondriacal delusional psychosis. I have tried the drug in a number of patients but found it singularly ineffective. Others have told me that they have had similar experience. It is possible that it has some effect but it is far from "well established" that it is "very effective". On the other hand it is well established that morbid jealousy in alcoholics frequently subsides when they stop drinking.

Department of Psychiatry

SAmuel I. Cohen

The London Hospital Medical College

Turner Street

London E1 2AD

\section{Syphilis screening}

SIR: Boodhoo (Journal, August 1989, 155, 259-262) poses a number of questions relating to syphilis screening in a psychogeriatric population.

Although syphilis remains a cause of reversible psychiatric illness, appropriate studies in patients 